\title{
NUMERICAL RANGE OF THE DERIVATION OF AN INDUCED OPERATOR
}

\section{RANDALL R. HOLMES, CHI-KWONG LI and TIN-YAU TAM ${ }^{\leftleftarrows}$}

(Received 15 April 2005; revised 3 April 2006)

Communicated by J. Du

\begin{abstract}
Let $V$ be an $n$-dimensional inner product space over $\mathbb{C}$, let $H$ be a subgroup of the symmetric group on $\{1, \ldots, m\}$, and let $\chi: H \rightarrow \mathbb{C}$ be an irreducible character. Denote by $V_{x}^{m}(H)$ the symmetry class of tensors over $V$ associated with $H$ and $\chi$. Let $K(T) \in \operatorname{End}\left(V_{\chi}^{m}(H)\right)$ be the operator induced by $T \in \operatorname{End}(V)$, and let $D_{K}(T)$ be the derivation operator of $T$. The decomposable numerical range $W^{*}\left(D_{K}(T)\right)$ of $D_{K}(T)$ is a subset of the classical numerical range $W\left(D_{K}(T)\right)$ of $D_{K}(T)$. It is shown that there is a closed star-shaped subset $\mathscr{S}$ of complex numbers such that

$$
\mathscr{S} \subseteq W^{*}\left(D_{K}(T)\right) \subseteq W\left(D_{K}(T)\right)=\operatorname{conv} \mathscr{S}
$$

where conv $\mathscr{S}$ denotes the convex hull of $\mathscr{S}$. In many cases, the set $\mathscr{S}$ is convex, and thus the set inclusions are actually equalities. Some consequences of the results and related topics are discussed.

2000 Mathematics subject classification: primary 15A69, 15 A42.

Keywords and phrases: symmetry class of tensors, numerical range, induced operator, derivation, convexity.
\end{abstract}

\section{Introduction}

Let $V$ be an $n$-dimensional inner product space over $\mathbb{C}$. Let $S_{m}$ be the symmetric group of degree $m$ on the set $\{1, \ldots, m\}$. Each $\sigma \in S_{m}$ gives rise to a linear operator $P(\sigma)$ on $\otimes^{m} V$ :

$$
P(\sigma)\left(v_{1} \otimes v_{2} \otimes \cdots \otimes v_{m}\right):=v_{\sigma^{-3}(1)} \otimes v_{\sigma^{-1}(2)} \otimes \cdots \otimes v_{\sigma^{-1}(m)}, \quad v_{1}, \ldots, v_{m} \in V .
$$

The second author is partially supported by a USA NSF grant and a HK RGC grant.

(C) 2007 Australian Mathematical Society 1446-7887/07 \$A2.00+0.00 
Let $H$ be a subgroup of $S_{m}$ and let $\chi: H \rightarrow \mathbb{C}$ be an irreducible character of $H$. The symmetrizer

$$
S_{\chi}:=\frac{\chi(e)}{|H|} \sum_{\sigma \in H} \chi(\sigma) P(\sigma) \in \operatorname{End}\left(\otimes^{m} V\right)
$$

is an orthoprojector with respect to the inner product on $\otimes^{m} V$ induced by the inner product on $V$ via

$$
\left(u_{1} \otimes \cdots \otimes u_{m}, v_{1} \otimes \cdots \otimes v_{m}\right):=\prod_{i=1}^{m}\left(u_{i}, v_{i}\right)
$$

The range of $S_{x}$

$$
V_{\chi}^{m}(H):=S_{\chi}\left(\otimes^{m} V\right)
$$

is called the symmetry class of tensors over $V$ associated with $H$ and $\chi$. The elements in $V_{x}^{m}(H)$ of the form $S_{\chi}\left(v_{1} \otimes \cdots \otimes v_{m}\right)$ are called decomposable symmetrized tensors and are denoted by $v_{1} * \cdots * v_{m}$.

Let $T \in \operatorname{End}(V)$. There is a unique induced operator $K(T)$ acting on $V_{\chi}^{m}(H)$ satisfying

$$
K(T) v_{1} * \cdots * v_{m}=T v_{1} * \cdots * T v_{m} .
$$

Furthermore, one can define the derivation operator $D_{K}(T)$ of $T$ by

$$
D_{K}(T)=\left.\frac{\mathrm{d}}{\mathrm{dt}} K(I+t T)\right|_{t=0},
$$

which acts on $V_{x}^{m}(H)$ in the following way:

$$
D_{K}(T) v_{1} * \cdots * v_{m}=\sum_{j=1}^{m} v_{1} * \cdots * v_{j-1} * T v_{j} * v_{j+1} * \cdots * v_{m}
$$

Clearly $T \mapsto D_{K}(T)$ is linear. See [16,17] for general background on $K(T)$ and $D_{K}(T)$.

Define the numerical range and the decomposable numerical range of a linear operator $L$ acting on $V_{x}^{m}(H)$ by

$$
W(L)=\left\{(L x, x): x \in V_{x}^{m}(H),(x, x)=1\right\},
$$

and

$$
W^{*}(L)=\left\{(L x, x): x \in V_{x}^{m}(H) \text { is decomposable, }(x, x)=1\right\},
$$

respectively. The numerical range and the decomposable numerical range are useful concepts for studying linear operators, and they also have applications to other areas such as quantum physics (see $[2,3,13-15,19]$ ). Clearly

$$
W^{*}(L) \subseteq W(L)
$$


However, since a unit vector in $V_{x}^{m}(H)$ need not be decomposable $[16,17,20,25]$, one cannot expect that the above set inclusion is equality in general. Nonetheless, in this paper we show that there is a closed star-shaped subset $\mathscr{S}$ of $\mathbb{C}$ such that

$$
\mathscr{S} \subseteq W^{*}\left(D_{K}(T)\right) \subseteq W\left(D_{K}(T)\right)=\operatorname{conv} \mathscr{S} .
$$

We give several examples for which $\mathscr{S}$ is actually convex so that all of the above sets are equal. For instance, this is the case when $H=S_{m}$ (see Theorem 4.2). In particular $\mathscr{S}=$ conv $\mathscr{S}$ when $\chi$ is the alternating character of $S_{m}$ with $m \leq n$. In this case $V_{x}^{m}(H)$ is the $m$ th exterior space $\wedge^{m} V$, which has the special feature that each unit decomposable vector $u_{1} * \cdots * u_{m}$ is equal to some $v_{1} * \cdots * v_{m}$ with $v_{1}, \ldots, v_{m}$ orthonormal vectors in $V$. Consequently, the decomposable numerical range $W^{*}\left(D_{K}(T)\right)$ of $D_{K}(T)$ is equal to the $m$ th higher numerical range of $T$ [19]

$$
W_{m}(T)=\left\{\sum_{j=1}^{m}\left(T v_{j}, v_{j}\right):\left\{v_{1}, \ldots, v_{m}\right\} \text { is an orthonormal set in } V\right\},
$$

which is convex by a result of Berger [19].

We present some preliminaries in Section 2, and prove the set inclusion result in Section 3. In Section 4 we discuss the situation where $H$ is the dihedral group, the alternating group, or the full symmetric group (or, more generally, a Young subgroup). Some consequences are deduced in Section 5 and related results are discussed in Section 6.

\section{Preliminaries}

In this section, we present some preliminary results for induced operators; see $[16,17,20,25]$ for general background.

Let $I(H)$ be the set of irreducible characters of $H \leq S_{m}$. If $\chi, \xi \in I(H)$ and $\chi \neq \xi$, then $S_{\chi} S_{\xi}=0$. Moreover $\sum_{\chi \in I(H)} S_{\chi}$ is the identity operator on $\otimes^{m} V$. So we have the orthogonal sum

$$
\otimes^{m} V=\dot{\sum}_{x \in I(H)} V_{x}^{m}(H)
$$

Let $\Gamma_{m, n}$ be the set of sequences $\alpha=(\alpha(1), \ldots, \alpha(m))$ with $1 \leq \alpha(j) \leq n$ for $j=1, \ldots, m$. Two sequences $\alpha$ and $\beta$ in $\Gamma_{m, n}$ are said to be equivalent modulo $H$, denoted by $\alpha \sim \beta$, if there exists $\sigma \in H$ such that $\beta=\alpha \sigma$, where $\alpha \sigma:=(\alpha(\sigma(1)), \ldots, \alpha(\sigma(m)))$. This equivalence relation partitions $\Gamma_{m, n}$ into equivalence classes. Let $\Delta$ be a system of representatives for the equivalence classes such 
that each sequence in $\Delta$ is first in its equivalence class relative to the lexicographic order. Define $\bar{\Delta}$ as the subset of $\Delta$ consisting of those sequences $\alpha \in \Delta$ such that

$$
\sum_{\sigma \in H_{\alpha}} \chi(\sigma) \neq 0
$$

where $H_{\alpha}:=\{\sigma \in H: \alpha \sigma=\alpha\}$ is the stabilizer subgroup of $\alpha$.

Let $\mathscr{B}=\left\{e_{1}, \ldots, e_{n}\right\}$ be a basis for $V$. Then $\left\{e_{\alpha}^{\otimes}:=e_{\alpha(1)} \otimes \cdots \otimes e_{\alpha(m)}: \alpha \in \Gamma_{m, n}\right\}$ is a basis for $\otimes^{m} V$. Let

$$
e_{\alpha}^{*}:=S_{\chi} e_{\alpha}^{\otimes}=\frac{\chi(e)}{|H|} \sum_{\sigma \in H} \chi(\sigma) e_{\alpha \sigma^{-1}(1)} \otimes \cdots \otimes e_{\alpha \sigma^{-1}(m)}
$$

for each $\alpha \in \Gamma_{m, n}$. Then $\left\{e_{\alpha}^{*}: \alpha \in \Gamma_{m, n}\right\}$ is a spanning set for the space $V_{x}^{m}(H)$, but it may not be linearly independent. Indeed some of these vectors may even be zero. It is known that $e_{\alpha}^{*} \neq 0$ if and only if the restriction of $\chi$ to $H_{\alpha}$ contains the principal character as an irreducible constituent [20, page 163]. Let

$$
\Omega:=\left\{\alpha \in \Gamma_{m, n}:(\chi, 1)_{H_{\alpha}} \neq 0\right\}
$$

Note that $\bar{\Delta}=\Delta \cap \Omega$ and

$$
\Omega=\bigcup_{\alpha \in \bar{\Delta}}\{\alpha \sigma: \sigma \in H\}
$$

see [20, Equation (6.15), page 164]. The set $\left\{e_{\alpha}^{*}: \alpha \in \Omega\right\}$ consists of the nonzero elements of $\left\{e_{\alpha}^{*}: \alpha \in \Gamma_{m, n}\right\}$. Moreover

$$
V_{\chi}^{m}(H)=\bigoplus_{\alpha \in \bar{\Delta}} O_{\alpha}
$$

where $O_{\alpha}:=\left\langle e_{\alpha \sigma}^{*}: \sigma \in H\right\rangle$ (called an orbital subspace). Freese's theorem [20, page 165 ] asserts that

$$
s_{\alpha}:=\operatorname{dim} O_{\alpha}=\frac{\chi(e)}{\left|H_{\alpha}\right|} \sum_{\sigma \in H_{\alpha}} \chi(\sigma)=\chi(e)(\chi, 1)_{H_{\alpha}} .
$$

We now construct a basis for $V_{x}^{m}(H)$. For each $\alpha \in \bar{\Delta}$, we find a basis for the orbital subspace $O_{\alpha}$ : choose a lexicographically ordered set $\left\{\alpha_{1}, \ldots, \alpha_{s_{\alpha}}\right\}$ from $\{\alpha \sigma: \sigma \in H\}$ such that $\left\{e_{\alpha_{1}}^{*}, \ldots, e_{\alpha_{s_{\alpha}}}^{*}\right\}$ is a basis for $O_{\alpha}$. Execute this procedure for each $\gamma \in \bar{\Delta}$. If $\{\alpha, \beta, \ldots\}$ is the lexicographically ordered set $\bar{\Delta}$, take

$$
\hat{\Delta}=\left\{\alpha_{1}, \ldots, \alpha_{s_{\alpha}}, \beta_{1}, \ldots, \beta_{s_{\beta}}, \ldots\right\}
$$


to be ordered as indicated. Then $\left\{e_{\alpha}^{*}: \alpha \in \hat{\Delta}\right\}$ is a basis for $V_{x}^{m}(H)$ (but the elements of $\hat{\Delta}$ need not be lexicographically ordered). Clearly $\bar{\Delta} \subseteq \hat{\Delta} \subseteq \Omega$. Although $\hat{\Delta}$ is not unique, it does not depend on the basis $\mathscr{B}$ since $\Delta$ and $\bar{\Delta}$ do not depend on $\mathscr{B}$. Thus if $\mathscr{B}^{\prime}=\left\{f_{1}, \ldots, f_{n}\right\}$ is another basis for $V$, then $\left\{f_{\alpha}^{*}: \alpha \in \hat{\Delta}\right\}$ is still a basis for $V_{x}^{m}(H)$.

The inner product of $V$ induces an inner product on $V_{x}^{m}(H)$ :

$$
\left(u^{*}, v^{*}\right)=\frac{\chi(e)}{|H|} \sum_{\sigma \in H} \chi(\sigma) \prod_{t=1}^{m}\left(u_{t}, v_{\sigma(t)}\right) .
$$

Let $\mathscr{B}=\left\{e_{1}, \ldots, e_{n}\right\}$ be an orthonormal basis for $V$. Then

and thus

$$
\left(e_{\alpha}^{*}, e_{\beta}^{*}\right)= \begin{cases}0 & \text { if } \alpha \neq \beta, \\ \frac{\chi(e)}{|H|} \sum_{\sigma \in H_{\alpha}} \chi(\sigma) & \text { if } \alpha=\beta,\end{cases}
$$

$$
\left\|e_{\alpha}^{*}\right\|^{2}=\frac{\chi(e)}{|H|} \sum_{\sigma \in H_{\alpha}} \chi(\sigma) .
$$

Hence (2.2) becomes $V_{x}^{m}(H)=\dot{\sum}_{\alpha \in \bar{\Delta}}\left\langle e_{\alpha \sigma}^{*}: \sigma \in H\right\rangle$, an orthogonal sum. However, those $e_{\alpha}^{*}$ 's of $\left\{e_{\alpha}^{*}: \alpha \in \hat{\Delta}\right\}$ belonging to the same orbital subspace need not be orthogonal.

It is known [25, page 103], and also follows from (2.3), that $\bar{\Delta}=\hat{\Delta}$ if and only if $\chi$ is linear. In such cases, $\left\{e_{\alpha}^{*}: \alpha \in \bar{\Delta}\right\}$ is an orthogonal basis for $V_{\chi}^{m}(H)$.

We give several common examples of symmetry classes of tensors and induced operators.

EXAMPLE 1. Assume $1 \leq m \leq n, H=S_{m}$, and $\chi$ is the alternating character, that is, $\chi(\sigma)=\operatorname{sgn}(\sigma)$. Then $V_{\chi}^{m}(\bar{H})$ is the $m$ th exterior space $\wedge^{m} V, \bar{\Delta}=\hat{\Delta}=Q_{m, n}$, the set of strictly increasing sequences in $\Gamma_{m, n}, \Delta=G_{m, n}$, the set of nondecreasing sequences in $\Gamma_{m, n}$, and $K(T)$ is the $m$ th compound of $T \in \operatorname{End}(V)$, usually denoted by $C_{m}(T)$.

EXAMPLE 2. Assume $H=S_{m}$ and $\chi \equiv 1$, the principal character. Then $V_{\chi}^{m}(H)$ is the $m$ th completely symmetric space over $V=\mathbb{C}^{n}, \bar{\Delta}=\hat{\Delta}=\Delta=G_{m, n}$, and $K(T)$ is the $m$ th induced power of $T \in \operatorname{End}(V)$, usually denoted by $P_{m}(T)$.

EXAMPLE 3 (see [13]). Assume $H=\{e\}$, where $e$ is the identity in $S_{m}$ (and $\chi \equiv 1$, which is the only irreducible character). Then $V_{\chi}^{m}(H)=\otimes^{m} V, \bar{\Delta}=\hat{\Delta}=\Delta=\Gamma_{m, n}$, and $K(T)=\otimes^{m} T$ is the $m$ th tensor power of $T \in \operatorname{End}(V)$.

We now provide an example with nonlinear irreducible character. 
EXAMPLE 4 (see [13]). Assume $H=S_{3}$ and $\chi=\chi_{3}$ (notation as in [12, page 157]), the only nonlinear irreducible character. We have $\chi(e)=2, \chi((12))=0$, $\chi((123))=-1$. Assume $n:=\operatorname{dim} V=2$. Then

$$
\bar{\Delta}=\{(1,1,2),(1,2,2)\}, \quad \hat{\Delta}=\{(1,1,2),(1,2,1),(1,2,2),(2,1,2)\} ;
$$

see [20, page 164]. Let $\mathscr{B}=\left\{e_{1}, e_{2}\right\}$ be a basis for $V$. Then

$$
\mathscr{B}^{*}=\left\{e_{(1,1,2)}^{*}, e_{(1,2,1)}^{*}, e_{(1,2,2)}^{*}, e_{(2,1,2)}^{*}\right\}
$$

is a basis for $V_{\chi}^{m}(H)$, and (see [25, pages 98-101])

$$
e_{(2,1,1)}^{*}=-e_{(1,1,2)}^{*}-e_{(1,2,1)}^{*}, \quad e_{(2,2,1)}^{*}=-e_{(1,2,2)}^{*}-e_{(2,1,2)}^{*} .
$$

Let $T \in \operatorname{End}(V)$ be defined by

$$
[T]_{\mathscr{B}}=\left(\begin{array}{ll}
a & b \\
c & d
\end{array}\right)
$$

By direct computation,

and

$$
[K(T)]_{\mathscr{B}^{*}}=\left(\begin{array}{cccc}
a^{2} d-a b c & 0 & a b d-b^{2} c & 0 \\
0 & a^{2} d-a b c & a b d-b^{2} c & b^{2} c-a b d \\
a c d-b c^{2} & 0 & a d^{2}-b c d & 0 \\
a c d-b c^{2} & b c^{2}-a c d & 0 & a d^{2}-b c d
\end{array}\right)
$$

$$
\left[D_{K}(T)\right]_{\mathscr{B}^{*}}=\left(\begin{array}{cccc}
2 a+d & 0 & b & 0 \\
0 & 2 a+d & b & -b \\
c & 0 & a+2 d & 0 \\
c & -c & 0 & a+2 d
\end{array}\right) .
$$

Observe that $\mathscr{B}^{*}$ is not an orthogonal basis even if $\mathscr{B}$ is an orthonormal basis, since

$$
\left(e_{(1,1,2)}^{*}, e_{(1,2,1)}^{*}\right)=\left(e_{(1,2.2)}^{*}, e_{(2.1,2)}^{*}\right)=-1 / 3 .
$$

Let $\mathbf{m}_{j}(\alpha)$ denote the number of occurrences of $j=1, \ldots, n$ in the sequence $\alpha \in \Gamma_{m, n}$. The vector $\mathbf{m}(\alpha)=\left(\mathbf{m}_{1}(\alpha), \ldots, \mathbf{m}_{n}(\alpha)\right)$ is called the multiplicity vector of $\alpha$.

Recall that $\Omega:=\left\{\alpha \in \Gamma_{m, n}:(\chi, 1)_{H_{a}} \neq 0\right\}$. Let $\mathbf{m}(\Omega):=\{\mathbf{m}(\alpha): \alpha \in \Omega\} \subseteq \mathbb{N}^{n}$ be the collection of multiplicity vectors of all $\alpha \in \Omega$. A vector $k \in \mathbb{N}^{m}$ is said to be admissible if $k \in \mathbf{m}(\Omega)$. We claim that $\mathbf{m}(\Omega)$ is invariant under the usual action of $S_{n}$ on $\mathbb{N}^{n}$ given by $k \tau=\left(k_{\tau(1)}, \ldots, k_{\tau(n)}\right)$ for $k \in \mathbb{N}^{n}, \tau \in S_{n}$. Let $\alpha \in \Omega$ and $\tau \in S_{n}$. With the definition $\tau \alpha=(\tau(\alpha(1)), \ldots, \tau(\alpha(m))$, we clearly have $H_{\tau \alpha}=H_{\alpha}$ so that $\tau \Omega=\Omega$. One easily checks that $\mathbf{m}(\alpha) \tau=\mathbf{m}\left(\tau^{-1} \alpha\right)$. Thus, $\mathbf{m}(\Omega) \tau=\mathbf{m}\left(\tau^{-1} \Omega\right)=\mathbf{m}(\Omega)$, as desired. 


\section{Set inclusion and convexity}

Let $T \in \operatorname{End}(V)$ have eigenvalues $\lambda_{1}(T), \ldots, \lambda_{n}(T)$ and let $\mathscr{B}=\left\{v_{1}, \ldots, v_{n}\right\}$ be an orthonormal basis of $V$. One calls $\mathscr{B}$ a Schur basis for $T$ if the matrix representation $A$ of $T$ with respect to $\mathscr{B}$ is upper triangular and $\operatorname{diag} A=\left(\lambda_{1}(T), \ldots, \lambda_{n}(T)\right)$. According to the Schur Triangularization Theorem, a Schur basis always exists no matter how the $\lambda_{i}(T)$ are preordered.

Assume that $\mathscr{B}$ is a Schur basis for $T$. The matrix of $D_{K}(T)$ relative to the basis $\left\{v_{\alpha}^{*}: \alpha \in \hat{\Delta}\right\}$ of $V_{x}^{m}(H)$ is upper triangular. Moreover, the diagonal entries of this matrix are $\lambda_{\alpha}=\sum_{j=1}^{n} m_{j}(\alpha) \lambda_{j}(T), \alpha \in \hat{\Delta}$. So, in particular, these are the eigenvalues of $D_{K}(T)$. We point out that it might not be possible to choose $\hat{\Delta}$ for which $\left\{v_{\alpha}^{*}: \alpha \in \hat{\Delta}\right\}$ is orthogonal $[8,9,26]$ and thus this need not be a Schur basis for $D_{K}(T)$.

Denote by $\operatorname{Spec}(L)$ the spectrum of a linear operator $L$. The following is an extension of the well-known result $\operatorname{Spec} L \subset W(L)$; see [7].

Proposition 3.1. Let $T \in \operatorname{End}(V)$. Then $\operatorname{Spec}\left(D_{K}(T)\right) \subset W^{*}\left(D_{K}(T)\right)$.

ProOf. Let $x_{1}, \ldots, x_{n} \in V$ and let $\alpha \in \Omega$. We have

$$
\begin{aligned}
\left(D_{K}(T) x_{\alpha}^{*}, x_{\alpha}^{*}\right) & =\left(D_{K}(T) x_{\alpha(1)} * \cdots * x_{\alpha(m)}, x_{\alpha(1)} * \cdots * x_{\alpha(m)}\right) \\
& =\left(\sum_{i=1}^{m} x_{\alpha(1)} * \cdots T x_{\alpha(i)} * \cdots x_{\alpha(m)}, x_{\alpha(1)} * \cdots * x_{\alpha(m)}\right) \\
& =\sum_{i=1}^{m} \frac{\chi(e)}{|H|} \sum_{\sigma \in H} \chi(\sigma)\left(T x_{\alpha(i)}, x_{\alpha \sigma(i)}\right) \prod_{j \neq i}\left(x_{\alpha(j)}, x_{\alpha \sigma(j)}\right) .
\end{aligned}
$$

Now suppose $\left\{x_{1}, \ldots, x_{n}\right\}$ is an orthonormal basis for $V$. Let $\sigma \in H$ and suppose that $\prod_{j \neq i}\left(x_{\alpha(j)}, x_{\alpha \sigma(j)}\right) \neq 0$. Then $\alpha(j)=\alpha \sigma(j)$ for all $j \neq i$. However since $\mathbf{m}_{t}(\alpha)=\mathbf{m}_{t}(\alpha \sigma)$ for $t=1, \ldots, n$, we also have $\alpha(i)=\alpha \sigma(i)$. So $\sigma \in H_{\alpha}$. Thus

$$
\begin{aligned}
\left(D_{K}(T) x_{\alpha}^{*}, x_{\alpha}^{*}\right) & =\sum_{i=1}^{m} \frac{\chi(e)}{|H|} \sum_{\sigma \in H_{\alpha}} \chi(\sigma)\left(T x_{\alpha(i)}, x_{\alpha(i)}\right) \\
& =\frac{\chi(e)}{|H|} \sum_{\sigma \in H_{\alpha}} \chi(\sigma) \sum_{i=1}^{m}\left(T x_{\alpha(i)}, x_{\alpha(i)}\right) \\
& =\left\|x_{\alpha}^{*}\right\|^{2} \sum_{t=1}^{n} m_{t}(\alpha)\left(T x_{t}, x_{t}\right) .
\end{aligned}
$$

Since $\left\|x_{\alpha}^{*}\right\|^{2}=(\chi(e) /|H|) \sum_{\sigma \in H_{\alpha}} \chi(\sigma)=(\chi, 1)_{H_{\alpha}} \neq 0$, the desired result follows if $\left\{x_{1}, \ldots, x_{n}\right\}$ is a Schur basis. 
Since $\operatorname{Re} W(L)=W(\operatorname{Re}(L))$ where Re denotes the real part of a complex number as well as the Hermitian part of an operator, the right vertical support line of $W(L)$ equals $\{\mu \in \mathbb{C}: \operatorname{Re} \mu=\max \operatorname{Spec}(\operatorname{Re} L)\}$.

Given $c=\left(c_{1}, \ldots, c_{n}\right) \in \mathbb{C}^{n}$, the $c$-numerical range of $T \in \operatorname{End}(V)$ is the following subset of $\mathbb{C}$ :

$$
W_{c}(T):=\left\{\sum_{j=1}^{n} c_{j}\left(T x_{j}, x_{j}\right):\left\{x_{1}, \ldots, x_{n}\right\} \text { is an orthonormal basis for } V\right\} .
$$

It is known that $W_{c}(T)$ is convex if $c \in \mathbb{R}^{n}$, but it can fail to be convex if $c \in \mathbb{C}^{n}$; see [27] (see [21] for another proof and [23] for a generalization in the context of compact Lie groups).

We say that $c=\left(c_{1}, \ldots, c_{n}\right) \in \mathbb{R}^{n}$ is majorized by $d=\left(d_{1}, \ldots, d_{n}\right) \in \mathbb{R}^{n}$, written $c \prec d$, if for each $1 \leq k \leq n$ the sum of the $k$ largest entries of $c$ is not larger than that of $d$, and $\sum_{j=1}^{n} c_{j}=\sum_{j=1}^{n} d_{j}$. Majorization induces a partial order on the set of orbits (under the entry permutation action of $S_{n}$ ) of those vectors in $\mathbb{R}^{n}$ having the same entry sum. Two vectors $x, y \in \mathbb{R}^{n}$ are said to be comparable if either $x \prec y$ or $y \prec x$. Clearly not all pairs of vectors are comparable; for example, $x=(4,1,1)$ and $y=(3,3,0)$ are not comparable.

It is known that if $c$ is majorized by $d$, then $W_{c}(T) \subseteq W_{d}(T)$; see $[1,6]$.

THEOREM 3.2. The set

$$
\mathscr{S}:=\bigcup_{\alpha \in \Omega} W_{\mathbf{m}(\alpha)}(T)=\bigcup_{\alpha \in \hat{\Delta}} W_{\mathbf{m}(\alpha)}(T)=\bigcup_{\alpha \in \bar{\Delta}} W_{\mathbf{m}(\alpha)}(T)
$$

is star-shaped with $(m / n)$ tr $T$ as a star-center. Moreover,

$$
\mathscr{S} \subseteq W^{*}\left(D_{K}(T)\right) \subseteq W\left(D_{K}(T)\right)=\operatorname{conv} \mathscr{S} .
$$

All these sets are equal to $W_{\mathbf{m}(\delta)}(T)$ if there is $\delta \in \Omega$ such that $\mathbf{m}(\delta)$ majorizes $\mathbf{m}(\alpha)$ for all $\alpha \in \Omega$ (respectively, $\alpha \in \bar{\Delta}$ ).

PROOF. Recall (2.1) $\Omega=\bigcup_{\alpha \in \Delta}\{\alpha \sigma: \sigma \in H\}$. Clearly $\mathbf{m}(\alpha)=\mathbf{m}(\alpha \sigma)$ and thus $W_{\mathbf{m}(\alpha)}(T)=W_{\mathbf{m}(\alpha \sigma)}(T)$ for any $\alpha \in \Gamma_{m, n}, \sigma \in S_{m}$. So $\mathscr{S}:=\bigcup_{\alpha \in \Omega} W_{\mathbf{m}(\alpha)}(T)=$ $\bigcup_{\alpha \in \bar{\Delta}} W_{\mathbf{m}(\alpha)}(T)$. Since $\Omega \supseteq \hat{\Delta} \supseteq \bar{\Delta}$, we have $\mathscr{S}=\bigcup_{\alpha \in \hat{\Delta}} W_{\mathbf{m}(\alpha)}(T)$ as well.

For any $\alpha \in \Omega$, we have $(m / n) \operatorname{tr} T \in W_{\mathbf{m}(\alpha)}(T)$. This is because there is an orthonormal basis $\left\{u_{1}, \ldots, u_{n}\right\}$ of $V$ such that $\left(T u_{i}, u_{i}\right)=(1 / n) \operatorname{tr} T$ for all $i=1, \ldots, n$ (see [5]), and $m=\sum_{t=1}^{n} \mathbf{m}_{t}(\alpha)$. Thus the set $\mathscr{S}$ is star-shaped with $(m / n) \operatorname{tr} T$ as a star-center by the convexity of each. $W_{\mathrm{m}(\alpha)}(T)$ for $\alpha \in \Omega$ (also see [24]).

Each element of $W_{\mathbf{m}(\alpha)}(T)$ is of the form $\sum_{t=1}^{n} m_{t}(\alpha)\left(T x_{i}, x_{t}\right)$ for some orthonormal basis $\left\{x_{1}, \ldots, x_{n}\right\}$. By (3.2),

$$
W_{\mathbf{m}(\alpha)}(T) \subseteq W^{*}\left(D_{K}(T)\right) .
$$


Hence

$$
\mathscr{S} \subseteq W^{*}\left(D_{K}(T)\right) \subseteq W\left(D_{K}(T)\right) .
$$

Next, we are going to prove that $W\left(D_{K}(T)\right)=\operatorname{conv} \mathscr{S}$. Since $W\left(D_{K}(T)\right)$ is convex [7, page 110] and contains $\mathscr{S}$, we get $W\left(D_{K}(T)\right) \supseteq$ conv $\mathscr{S}$. Therefore, it remains to prove the other inclusion, and for this it suffices to prove that every extreme point of $W\left(D_{K}(T)\right)$ belongs to $W_{\mathrm{m}(\alpha)}(T)$ for some $\alpha \in \hat{\Delta}$.

First, we consider an exposed boundary point $\mu \in W\left(D_{K}(T)\right)$. By definition, there exists a support line of $W\left(D_{K}(T)\right)$ intersecting $W\left(D_{K}(T)\right)$ at the point $\mu$ alone. Then there is $r \in[0,2 \pi)$ such that $e^{i r} \mu$ is the only point of $W\left(e^{i r} D_{K}(T)\right)=e^{i r} W\left(D_{K}(T)\right)$ on the right vertical support line of $W\left(e^{i r} D_{K}(T)\right)$. Since $T \mapsto D_{K}(T)$ is linear, $D_{K}\left(\operatorname{Re}\left(e^{i r} T\right)\right)=\operatorname{Re}\left(e^{i r} D_{K}(T)\right)$. By the discussion before the theorem,

$$
\begin{aligned}
\operatorname{Re} e^{i r} \mu & =\max \operatorname{Spec}\left(\operatorname{Re}\left(e^{i r} D_{K}(T)\right)\right)=\max \operatorname{Spec}\left(D_{K}\left(\operatorname{Re}\left(e^{i r} T\right)\right)\right) \\
& =\max _{\alpha \in \hat{\Delta}} \sum_{j=1}^{n} m_{j}(\alpha) \lambda_{j}\left(\operatorname{Re}\left(e^{i r}(T)\right)\right) .
\end{aligned}
$$

Let $\left\{v_{1}, \ldots, v_{n}\right\}$ be an orthonormal basis of eigenvectors corresponding to the eigenvalues $\lambda_{1}\left(\operatorname{Re}\left(e^{i r} T\right)\right) \geq \cdots \geq \lambda_{n}\left(\operatorname{Re}\left(e^{i r} T\right)\right)$ of the Hermitian operator $\operatorname{Re}\left(e^{i r} T\right)$. Then

$$
\lambda_{j}\left(\operatorname{Re}\left(e^{i r} T\right)\right)=\left(\operatorname{Re}\left(e^{i r} T\right) v_{j}, v_{j}\right)=\operatorname{Re}\left(e^{i r} T v_{j}, v_{j}\right), \quad j=1, \ldots, n .
$$

So there is $\alpha \in \hat{\Delta}$ such that

$$
\operatorname{Re} e^{i r} \mu=\operatorname{Re} \sum_{j=1}^{n} m_{j}(\alpha)\left(e^{i r} T v_{j}, v_{j}\right)
$$

Hence, the point

$$
\sum_{j=1}^{n} m_{j}(\alpha)\left(e^{i r} T v_{j}, v_{j}\right) \in W_{\mathbf{m}(\alpha)}\left(e^{i r} T\right) \subseteq W\left(D_{K}\left(e^{i r} T\right)\right)=W\left(e^{i r} D_{K}(T)\right)
$$

lies on the right vertical support line of $W\left(e^{i r} D_{K}(T)\right)$. Since $e^{i r} \mu$ is the only point of $W\left(e^{i r} D_{K}(T)\right)$ lying on the right vertical support line, $e^{i r} \mu=\sum_{j=1}^{n} m_{j}(\alpha)\left(e^{i r} T v_{j}, v_{j}\right)$ and hence $\mu=\sum_{j=1}^{n} m_{j}(\alpha)\left(T v_{j}, v_{j}\right) \in W_{\mathbf{m}(\alpha)}(T)$.

Straszewicz's Theorem [22, page 167] asserts that the set of exposed points of any closed convex set $C$ is a dense subset of the set of extreme points of $C$. The convex hull of two circular disks in $\mathbb{C}$ of the same radius, but of different centers, is a simple example for seeing how an extreme point is the limit of a sequence of exposed points. Now $W_{\mathrm{m}(\alpha)}(T) \subset \mathbb{C}, \alpha \in \hat{\Delta}$, are finitely many closed sets. So $\mathscr{S}=\bigcup_{\alpha \in \hat{\Delta}} W_{\mathbf{m}(\alpha)}(T)$ is closed and contains all the exposed points of the closed convex set $W\left(D_{K}(T)\right)$. We conclude that every extreme point of $W\left(D_{K}(T)\right)$ belongs to $\mathscr{S}$. 
Finally, suppose there is $\delta \in \Omega$ such that $\mathbf{m}(\delta)$ majorizes $\mathbf{m}(\alpha)$ for all $\alpha \in \Omega$ (respectively, $\alpha \in \bar{\Delta}$ ). Then $W_{\mathbf{m}(\delta)}(T) \supseteq W_{\mathbf{m}(\alpha)}(T)$ for each $\alpha \in \Omega$ (respectively, $\alpha \in \bar{\Delta})$, whence $\mathscr{S}=W_{\mathbf{m}(\delta)}(T)$. Since $W_{\mathbf{m}(\delta)}(T)$ is convex, we have conv $\mathscr{S}=\mathscr{S}$ and so the inclusions in the statement of the theorem are all equalities. The last assertion of the theorem follows.

\section{Largest multiplicity vector and convexity}

A vector $x \in \mathbf{m}(\Omega)$ is said to be a largest vector of $\mathbf{m}(\Omega)$ if $y \prec x$ for all $y \in \mathbf{m}(\Omega)$. A largest vector of $\mathbf{m}(\Omega)$, if it exists, is unique up to permutation of its entries.

In this section, we give examples for which the set equalities

$$
\mathscr{S}=W^{*}\left(D_{K}(T)\right)=W\left(D_{K}(T)\right)=\operatorname{conv} \mathscr{S}
$$

hold. We establish the indicated equalities in each case by showing that either $\Omega$ is empty, so that these sets are all empty as well, or $\mathbf{m}(\Omega)$ has a largest vector $k$, so that these sets all equal $W_{k}(T)$ by Theorem 3.2. We also give examples to illustrate that some of these equalities can fail to hold (see Theorems 4.5 and 4.6). We point out that in all of the examples in Section 2 there is a largest vector of $\mathbf{m}(\Omega)$, so (4.1) holds in those cases.

Our first example was already observed in [3, Theorem 8.1].

PROPOSITION 4.1. Suppose $\chi$ is the principal character of the subgroup $H$ of $S_{m}$. Then $\mathbf{m}(\Omega)$ has a largest vector, namely $(m, 0, \ldots, 0)$, and so (4.1) holds. Moreover the sets in (4.1) all equal $m W(T)$.

PROOF. As observed in [3, Theorem 8.1], $(m, 0, \ldots, 0)=\mathbf{m}(\delta)$, where $\delta=$ $(1,1, \ldots, 1) \in \Omega$, so $(m, 0, \ldots, 0)$ is in $\mathbf{m}(\Omega)$, and it is clearly a largest vector of $\mathbf{m}(\Omega)$. Therefore, Theorem 3.2 states that the sets in (4.1) all equal $W_{\mathbf{m}(\delta)}(T)=$ $\{m(T x, x): x \in V,(x, x)=1\}$.

THEOREM 4.2. If $H=S_{m}$, then (4.1) holds.

Proof. Assume $H=S_{m}$ and let $\pi_{x}$ be the partition of $m$ corresponding to $\chi$ [20]. If $\pi_{x}$ has greater than $n$ parts, then $\Omega$ is empty [20, Corollary 6.38, page 169]. Assume $\pi_{\chi}$ has at most $n$ parts, so that, in particular, it is a multiplicity vector. It is known that a multiplicity vector $k \in \mathbb{N}^{n}$ is admissible if and only if $k$ is majorized by $\pi_{x}$ [13], [20, page 169]. It follows that $\pi_{x}$ is a largest vector of $\mathbf{m}(\Omega)$. 
For a partition $\mu=\left(\mu_{1}, \ldots, \mu_{r}\right)$ of $m$, the corresponding Young subgroup of $S_{m}$ is the internal direct product $S_{\mu}=S_{M_{1}} \times \cdots \times S_{M_{r}}$, where $S_{M_{i}}$ is the subgroup of $S_{\mathrm{m}}$ consisting of those permutations that fix every integer not contained in the set

$$
M_{i}:=\left\{1 \leq k \leq m: \sum_{j=1}^{i-1} \mu_{j}<k \leq \sum_{j=1}^{i} \mu_{j}\right\}
$$

(an empty sum being interpreted as zero).

THEOREM 4.3. Let $\mu=\left(\mu_{1}, \ldots, \mu_{r}\right)$ be a partition of $m$. If $H=S_{\mu}$ (Young subgroup), then (4.1) holds.

PROOF. Assume $H=S_{\mu}=\prod_{i=1}^{r} S_{M_{i}}$ (notation as above). Then $\chi=\prod_{i=1}^{r} \chi_{i}$ with $\chi_{i}$ an irreducible character of $S_{M_{i}}(1 \leq i \leq r)$.

Denoting by $\Gamma_{M_{i}, n}$ the set of functions from $M_{i}$ to $\{1, \ldots, n\}$, we have a bijection $\Gamma_{m, n} \rightarrow \prod_{i=1}^{r} \Gamma_{M_{i}, n}$ given by $\alpha \mapsto\left(\alpha_{1}, \ldots, \alpha_{r}\right)$, where $\alpha_{i}$ denotes the restriction of $\alpha$ to $M_{i}$. For $\alpha \in \Gamma_{m, n}$ we have $H_{\alpha}=\prod_{i=1}^{r}\left(S_{M_{i}}\right)_{\alpha_{i}}$ so that

$$
(\chi, 1)_{H_{\alpha}}=\prod_{i=1}^{r}\left(\chi_{i}, 1\right)_{\left(S_{M_{i}}\right)_{\alpha_{i}}} .
$$

Therefore, $\alpha \in \Omega$ if and only if $\alpha_{i} \in \Omega_{i}:=\left\{\beta \in \Gamma_{M_{i}, n}:\left(\chi_{i}, 1\right)_{\left(S_{M_{i}}\right)_{\beta}} \neq 0\right\}$ for each $i$. Clearly $\mathbf{m}(\alpha)=\sum_{i} \mathbf{m}\left(\alpha_{i}\right)\left(\alpha \in \Gamma_{m, n}\right)$, so it follows from the observations above that $\mathbf{m}(\Omega)=\sum_{i} \mathbf{m}\left(\Omega_{i}\right)$. Identifying $S_{M_{i}}$ with $S_{\mu_{i}}$ in the natural way, we have that $\chi_{i}$ equals $\chi_{\pi_{i}}$ for some partition $\pi_{i}$ of $\mu_{i}$. If some $\pi_{i}$ has greater than $\mu_{i}$ parts, then $\Omega_{i}$ is empty, whence $\Omega$ is empty and (4.1) holds. Assume each $\pi_{i}$ has at most $\mu_{i}$ parts. Then the proof of Theorem 4.2 shows that $\pi_{i}$ is a largest vector of $\mathbf{m}\left(\Omega_{i}\right)$ and thus $\pi:=\sum_{i} \pi_{i}$ is a largest vector of $\mathbf{m}(\Omega)$.

PROPOSITION 4.4. If $m \leq 5$, then (4.1) holds.

PROOF. Assume $m \leq 5$. Then majorization is a total order on the set of partitions of $m$. Since each vector in $\mathbf{m}(\Omega)$ lies in the orbit (under the entry permutation action of $S_{n}$ ) of some partition of $m$, it follows that $\mathbf{m}(\Omega)$ either is empty or has a largest vector.

In each of the examples so far, $\mathbf{m}(\Omega)$ has had a largest vector (or it has been empty). We next give an example for which $\mathbf{m}(\Omega)$ is nonempty and has no largest vector.

Assume $H=D_{m}(m \geq 3)$, the dihedral group, which is generated by

$$
r=\left(\begin{array}{ccccc}
1 & 2 & \cdots & m-1 & m \\
2 & 3 & \cdots & m & 1
\end{array}\right) \text { and } s=\left(\begin{array}{cccccc}
1 & 2 & 3 & \cdots & m-1 & m \\
1 & m & m-1 & \cdots & 3 & 2
\end{array}\right) .
$$


If $m$ is even, there are 4 irreducible characters of degree 1 , given by the following table:

$$
\begin{array}{rcrcl} 
& \psi_{1} & \psi_{2} & \psi_{3} & \psi_{4} \\
r^{k} & 1 & 1 & (-1)^{k} & (-1)^{k} \\
s r^{k} & 1 & -1 & (-1)^{k} & (-1)^{k+1}
\end{array}
$$

If $m$ is odd, then $\psi_{1}$ and $\psi_{2}$ are the only irreducible characters of degree 1. The other irreducible characters of $D_{m}$ are of degree 2 and are induced by certain irreducible characters of the cyclic subgroup $C_{m}=\langle r\rangle$.

THEOREM 4.5. Assume $H=D_{m}$ and let the notation be as above.

(1) Assume $\chi=\psi_{2}, m>5$, and $n \geq 3$. Then $\mathbf{m}(\Omega)$ contains $(m-2,1,1,0, \ldots, 0)$ and $(m-3,3,0, \ldots, 0)$ and each vector in $\mathbf{m}(\Omega)$ is majorized by one of these vectors. In particular, there is no largest admissible vector. In fact, there exists $T \in E$ Ed $(V)$ for which $\mathscr{S}$ is not convex and hence for which (4.1) fails to hold.

(2) If $\chi \neq \psi_{2}$ or $m \leq 5$ or $n<3$, then (4.1) holds.

ProOF. We first prove (1). We claim that $(m-1,1,0, \ldots, 0)$ is not admissible. Otherwise there is a $\gamma \in \Omega$ such that $\mathbf{m}(\gamma)=(m-1,1,0, \ldots, 0)$. We may assume $\gamma=(2,1, \ldots, 1)$ since $r \in D_{m}$. However now

$$
(\chi, 1)_{H_{\gamma}}=[\chi(1)+\chi(s)] / 2=(1-1) / 2=0,
$$

contradicting that $\gamma \in \Omega$.

We next claim that $(m-2,2,0, \ldots, 0)$ is not admissible. Otherwise there is a $\beta \in \Omega$ such that $\mathbf{m}(\beta)=(m-2,2,0, \ldots, 0)$. We may assume that $\beta=$ $(2,1, \ldots, 1,2,1, \ldots, 1)$, where the second 2 is in the $i$ th position for some $i \neq 1$. Then $H_{\beta}=\left\{e, s r^{m-i+1}\right\}$ and $(\chi, 1)_{H_{\beta}}=(1-1) / 2=0$, a contradiction.

Let $\delta=(3,2,1, \ldots, 1)$. Then $H_{\delta}=\{e\}$ and thus $(\chi, 1)_{H_{\delta}}=1$. So $(m-2,1$, $1,0, \ldots, 0)=\mathbf{m}(\delta)$ is admissible.

Let $\xi=(2,2,1,2,1, \ldots, 1)$. Any element of $H_{\xi}$ must fix 3 , since this is the only position $i$ for which $\xi_{i}=1$ and $\xi_{i-1}=2=\xi_{i+1}$ (subscripts modulo $m$ ), so that $H_{\xi} \subseteq\left\{e, s r^{m-4}\right\}$. However $s r^{m-4}(1)=5$, so $H_{\xi}=\{e\}$. Thus $(\chi, 1)_{H_{\xi}}=1$ and $(m-3,3,0, \ldots, 0)=\mathbf{m}(\xi)$ is admissible.

Using these observations, we see that all possible admissible vectors are majorized by either $\mathbf{m}(\delta)$ or $\mathbf{m}(\xi)$ and, since these two vectors are admissible and not comparable, we conclude that there is no largest admissible vector. Moreover, since $W_{c}(T) \subseteq W_{d}(T)$ whenever $c \prec d$, we find that

$$
\mathscr{S}:=\bigcup_{\alpha \in \Omega} W_{\mathbf{m}(\alpha)}(T)=W_{\mathbf{m}(\delta)}(T) \cup W_{\mathbf{m}(\xi)}(T) .
$$


Suppose $T=\operatorname{diag}(2+i, 1) \oplus 0_{n-2}$. It is a normal operator acting on $V=\mathbb{C}^{n}$. Let $t:=\left(t_{1}, \ldots, t_{n}\right)=(2+i, 1,0, \ldots, 0)$. Then (for example, see [18])

$$
W_{c}(T)=\operatorname{conv}\left\{c_{\sigma}^{T} t: \sigma \in S_{n}\right\}
$$

where $c_{\sigma}=\left(c_{\sigma(1)}, \ldots, c_{\sigma(n)}\right)$ for real $c \in \mathbb{R}^{n}$. So

and

$$
W_{\mathbf{m}(\delta)}(T)=\operatorname{conv}\left\{\begin{array}{l|l}
(m-2) t_{u}+t_{v}+t_{w} & \begin{array}{l}
\{u, v, w\} \text { is a three-element } \\
\text { subset of }\{1, \ldots, n\}
\end{array}
\end{array}\right\},
$$

$$
W_{\mathbf{m}(\xi)}(T)=\operatorname{conv}\left\{\begin{array}{l|l}
(m-3) t_{u}+3 t_{v} & \begin{array}{l}
\{u, v\} \text { is a two-element } \\
\text { subset of }\{1, \ldots, n\}
\end{array}
\end{array}\right\}
$$

In particular, $z_{1}=(2 m-3)+(m-2) i$ is a vertex of $W_{\mathbf{m}(\delta)}(T)$ and $z_{2}=(2 m-3)$ $+(m-3) i$ is a vertex of $W_{\mathbf{m}(\xi)}(T)$. However $z_{1}$ is the only vertex of $W_{\mathbf{m}(\delta)}(T)$ with real part $2 m-3$ and clearly $2 m-3$ is the largest possible among the real parts of vertices of $W_{\mathbf{m}(\delta)}(T)$. A similar statement holds for $z_{2}$ and $W_{\mathbf{m}(\xi)}(T)$. Therefore $\left(z_{1}+z_{2}\right) / 2=(2 m-3)+(m-5 / 2) i$ is a point on the segment joining $z_{1}$ and $z_{2}$ that does not belong to $W_{\mathbf{m}(\delta)}(T) \cup W_{\mathbf{m}(\xi)}(T)$. We conclude that $\mathscr{S}$ is not convex.

Now we prove (2). If $m \leq 5$, then the claim follows from Proposition 4.4. Assume $\chi \neq \psi_{2}$ and $m>5$. We consider cases:

(a) ( $\chi$ is the principal character). This case is handled by Proposition 4.1.

In the remaining cases, $\Omega$ is empty if $n=1$, in which case (4.1) holds. So we assume $n>1$.

(b) ( $\chi$ is of degree 2). Since $\chi$ is induced from a character of the normal subgroup $C_{m}$ of $H$, it vanishes on the complement of $C_{m}$. Let $\gamma=(2,1, \ldots, 1)$. Then clearly $H_{\gamma}=\{e, s\}$ and hence $(\chi, 1)_{H_{\gamma}}=1$. Thus $(m-1,1,0, \ldots, 0)=\mathbf{m}(\gamma)$ is admissible and it is clearly a largest such vector. Our claim now follows from Theorem 3.2 as usual.

(c) $\left(\chi=\psi_{3}\right)$. Let $\gamma=(2,1, \ldots, 1)$ as before. Then $(\chi, 1)_{H_{y}}=[\chi(1)+\chi(s)] / 2=$ $(1+1) / 2=1$. Thus, $(m-1,1,0, \ldots, 0)=\mathbf{m}(\gamma)$ is admissible and it is a largest such vector.

(d) $\left(\chi=\psi_{4}\right)$. First, $(m-1,1,0, \ldots, 0)$ is not admissible by an argument similar to that in the proof of (1). Let $\beta=(2,2,1, \ldots, 1)$. Then $H_{\beta}=\left\{e, s r^{m-1}\right\}$ and $(\chi, 1)_{H_{\beta}}=\left(1+(-1)^{m}\right) / 2=1$, using that $m$ must be even in this case. Thus, $(m-2,2,0, \ldots, 0)=\mathbf{m}(\beta)$ is a largest admissible vector.

Finally, assume $n<3, \chi=\psi_{2}$, and $m>5$. Since $n<3,(m-2,1,1,0, \ldots, 0)$ cannot be admissible. Therefore, in view of the proof of (1), we have that $(m-3,3$, $0, \ldots, 0)=\mathbf{m}(\xi)$ is a largest admissible vector, where $\xi=(2,2,1,2,1, \ldots, 1)$. This completes the proof. 
For the $T$ in the proof of part (1) of the theorem, we know only that one of the first two equalities of (4.1) fails to hold, in view of Theorem 3.2. That is, either $\mathscr{S} \neq W^{*}\left(D_{K}(T)\right)$ or $W^{*}\left(D_{K}(T)\right) \neq W\left(D_{K}(T)\right)$. It would be interesting to determine whether the line segment joining $z_{1}$ and $z_{2}$ in the above proof belongs to $W^{*}\left(D_{K}(T)\right)$, since an answer in the negative would imply that $W^{*}\left(D_{K}(T)\right) \neq W\left(D_{K}(T)\right)$.

Assume $H=A_{m}(m \geq 2)$, the alternating group. Suppose the irreducible character $\chi$ of $H$ is invariant under the conjugation action of $S_{m}$, that is, ${ }^{\sigma} \chi=\chi$ for all $\sigma \in S_{m}$, where ${ }^{\sigma} \chi(\tau)=\chi\left(\tau^{\sigma}\right)=\chi\left(\sigma^{-1} \tau \sigma\right)(\tau \in H)$. Then $\chi^{S_{m}}=\chi_{\pi}+\chi_{\pi^{\prime}}$ for some partition $\pi$ of $m$ (written $\pi \vdash m$ ) with $\pi^{\prime} \neq \pi$, where $\pi^{\prime}$ denotes the conjugate partition of $\pi$ (see $[10,(6.20),(6.17)]$ and $[20$, Theorem 4.47]).

Now suppose $\chi$ is not invariant. Then $\chi^{S_{m}}=\chi_{\pi}$ for some $\pi \vdash m$ with $\pi^{\prime}=\pi$. Moreover, $\left({ }^{\sigma} \chi\right)^{S_{m}}=\chi_{\pi}$ for every $\sigma \in S_{m}$ (see [10, (6.11)] and [20, Corollary 4.48]).

In summary, there exists $\pi \vdash m$ such that for every $\sigma \in S_{m}$,

$$
\left({ }^{\sigma} \chi\right)^{S_{m}}=2^{-t}\left(\chi_{\pi}+\chi_{\pi^{\prime}}\right)
$$

where

$$
\iota= \begin{cases}0, & \pi^{\prime} \neq \pi, \\ 1, & \pi^{\prime}=\pi .\end{cases}
$$

Note that $\iota$ is 0 or 1 according to whether $\chi$ is or is not invariant. By Frobenius Reciprocity, $\pi$ may be taken to be any partition of $m$ for which $\chi$ is a constituent of the restriction of $\chi_{\pi}$ to $H$.

THEOREM 4.6. Assume $H$ is the alternating group $A_{m}$ with $n:=\operatorname{dim} V \geq m \geq 2$, and let $\pi$ and $\pi^{\prime}$ be described as above.

(1) Then $\mathscr{S}=W_{\pi}(T) \cup W_{\pi^{\prime}}(T)$.

(2) If $\pi$ and $\pi^{\prime}$ are comparable, then (4.1) holds. In particular, if $\chi$ is not invariant, then (4.1) holds.

PROOF. We begin by establishing a formula. Let $x \vdash m$ and assume $x \neq\left[1^{m}\right]$. Then $S_{x}$ (Young subgroup) contains an odd permutation, whence $H S_{x}=S_{m}$. Therefore, Mackey's Subgroup Theorem [10, page 74] states $\left(\left(1_{S_{x}}\right)^{S_{m}}\right)_{H}=\left(1_{S_{x} \cap H}\right)^{H}$. Using this observation together with Frobenius Reciprocity and linearity of the inner product, we obtain for each $\sigma \in S_{m}$

$$
\begin{aligned}
2^{-\imath}\left[\left(\chi_{\pi},\left(1_{S_{x}}\right)^{S_{m}}\right)_{S_{m}}+\left(\chi_{\pi^{\prime}},\left(1_{S_{x}}\right)^{S_{m}}\right)_{S_{m}}\right] & \left.=\left({ }^{\sigma} \chi\right)^{S_{m}},\left(1_{S_{x}}\right)^{S_{m}}\right)_{S_{m}}=\left({ }^{\sigma} \chi,\left(1_{S_{x}}\right)^{S_{m}}\right)_{H} \\
& =\left({ }^{\sigma} \chi,\left(1_{S_{x} \cap H}\right)^{H}\right)_{H}=\left({ }^{\sigma} \chi, 1\right)_{S_{x} \cap H} .
\end{aligned}
$$

Next, we claim that $\mathbf{m}(\Omega)=\left\{x \in \mathbb{N}^{n}: x \prec \pi\right.$ or $\left.x \prec \pi^{\prime}\right\}$. Put

$$
M=\left\{x \tau: x \vdash m, \tau \in S_{n} \text {, and either } x \prec \pi \text { or } x \prec \pi^{\prime}\right\} .
$$


Since $n \geq m$ and $x \vdash m$ has at most $m$ parts, one can view $x$ as an element of $\mathbb{N}^{n}$ so that the expression $x \tau=\left(x_{\tau(1)}, \ldots, x_{\tau(n)}\right)\left(\tau \in S_{n}\right)$ is defined. According to our definition of majorization, we have $M=\left\{x \in \mathbb{N}^{n}: x \prec \pi\right.$ or $\left.x \prec \pi^{\prime}\right\}$. So it suffices to show that $\mathbf{m}(\Omega)=M$.

Let $x_{1} \in \mathbf{m}(\Omega)$. Then $x_{1}=\mathbf{m}\left(\alpha_{1}\right)$ for some $\alpha_{1} \in \Omega$. Now $x:=x_{1} \tau^{-1} \vdash m$ for some $\tau \in S_{n}$. We have $x=\mathbf{m}\left(\alpha_{1}\right) \tau^{-1}=\mathbf{m}\left(\tau \alpha_{1}\right)=\mathbf{m}(\alpha)$, where $\alpha=\tau \alpha_{1} \in \Omega$. Since the stabilizer in $S_{m}$ of $\alpha$ is conjugate to $S_{x}$, it follows that $H_{\alpha}=\left(S_{x}\right)^{\sigma} \cap H=\left(S_{x} \cap H\right)^{\sigma}$ for some $\sigma \in S_{m}$. Then

$$
\left({ }^{\sigma} \chi, 1\right)_{S_{x} \cap H}=(\chi, 1)_{\left(S_{x} \cap H\right)^{\sigma}}=(\chi, 1)_{H_{\alpha}} \neq 0 .
$$

If $x \neq\left[1^{m}\right]$, then the computation above implies that either $\left(\chi_{\pi},\left(1_{S_{x}}\right)^{s_{m}}\right)_{s_{m}}$ or $\left(\chi_{\pi^{\prime}},\left(1_{S_{x}}\right)^{S_{m}}\right)_{S_{m}}$ is nonzero (because each of them is a nonnegative integer) so that $x \prec \pi$ or $x \prec \pi^{\prime}$; see [11]. Since $\left[1^{m}\right] \prec \pi$, it follows that in all cases $x \prec \pi$ or $x \prec \pi^{\prime}$. Thus $x_{1} \in M$.

Now let $x_{1} \in M$. Then $x_{1}=x \tau$ for some $x \vdash m$ and $\tau \in S_{n}$ with $x \prec \pi$ or $x \prec \pi^{\prime}$. Set $\alpha=\left(1^{x_{1}}, 2^{x_{2}}, \ldots, t^{x_{t}}\right)$, where $t$ is the length of $x$. Note that $\alpha$ is in $\Gamma_{m, n}$ by the assumption $n \geq m$ and since $t \leq m$. Then $H_{\alpha}=S_{x} \cap H$. Assume $x \neq\left[1^{m}\right]$. Since $x \prec \pi$ or $x \prec \pi^{\prime}$, we see (by [11]) that the first member in the computation above (with $\sigma=1$ ) is nonzero. Thus $(\chi, 1)_{H_{\alpha}} \neq 0$, implying $\alpha \in \Omega$. If $x=\left[1^{m}\right]$, then $\alpha=(1,2, \ldots, m)$, so $H_{\alpha}=\{e\}$, whence $\alpha \in \Omega$. Thus, in all cases $x=\mathbf{m}(\alpha) \in \mathbf{m}(\Omega)$. Finally, $x_{1}=\mathbf{m}(\alpha) \tau=\mathbf{m}\left(\tau^{-1} \alpha\right) \in \mathbf{m}(\Omega)$ as desired.

Now we prove (1). Since $W_{c}(T) \subseteq W_{d}(T)$ whenever $c \prec d$, we have

$$
\mathscr{S}:=\bigcup_{\alpha \in \Omega} W_{\mathbf{m}(\alpha)}(T)=W_{\pi}(T) \cup W_{\pi^{\prime}}(T) .
$$

Finally, we prove (2). If $\pi \succ \pi^{\prime}$, then $\pi$ is a largest vector in $\mathbf{m}(\Omega)$ and (4.1) follows from Theorem 3.2. The same holds with $\pi$ and $\pi^{\prime}$ exchanged. Finally, if $\chi$ is not invariant, then $\pi^{\prime}=\pi$, so $\pi$ and $\pi^{\prime}$ are comparable and the proof is complete.

If $\pi=\left[5,2^{3}\right] \vdash 11$, then $\pi^{\prime}=\left[4^{2}, 1^{3}\right]$ so that $\pi$ and $\pi^{\prime}$ are not comparable and there is no largest admissible vector (see proof of theorem). It is easy to check that 11 is the least $m$ for which there exists a partition of $m$ not comparable to its conjugate.

\section{Some consequences}

Using Theorem 3.2, we extend some results on the classical numerical range to the decomposable numerical range. The irreducible character $\chi$ is said to be of determinant type [13] if $K(S)=(\operatorname{det} S)^{m / n} I$ for all $S \in \operatorname{End}(V)$, or equivalently, each $\alpha \in \bar{\Delta}$ satisfies $\mathbf{m}_{1}(\alpha)=\cdots=\mathbf{m}_{n}(\alpha)$ (in which case each $\mathbf{m}_{j}(\alpha)=m / n$ ). In 
this case, $D_{K}(T)=(m / n)(\operatorname{tr} T) l$, and $W^{*}\left(D_{K}(T)\right)=W\left(D_{K}(T)\right)=\{(m / n)(\operatorname{tr} T)\}$, which does not convey much information about $T$. Otherwise, we have the following.

THEOREM 5.1. Suppose $\chi$ is not of determinant type. Then $D_{K}(T)$ is scalar (respectively, Hermitian) if and only if $T$ is scalar (respectively, Hermitian). Consequently,

(a) $W^{*}\left(D_{K}(T)\right)$ or $W\left(D_{K}(T)\right)$ equals $\{\mu\}$ if and only if $T=(\mu / m) l$;

(b) $W^{*}\left(D_{K}(T)\right)$ or $W\left(D_{K}(T)\right)$ is a subset of $\mathbb{R}$ if and only if $T=T^{*}$.

PROOF. If $T$ is scalar or Hermitian, then clearly $D_{K}(T)$ has the corresponding property. Suppose $D_{K}(T)=\mu \mathrm{l}$. We claim that both $\operatorname{Re} T$ and $\operatorname{Im} T$ are scalar operators. If $\operatorname{Re} T$ were not a scalar operator, then the spectrum of $\operatorname{Re} D_{K}(T)=$ $D_{K}(\operatorname{Re} T)$ would not be a singleton set. It is because it contains $\sum_{j=1}^{n} m_{j}(\alpha) \lambda_{j}(\operatorname{Re} T)$, $\alpha \in \hat{\Delta}$. Since $\chi$ is not of determinant type, we may assume that $\mathbf{m}_{1}(\alpha)>\mathbf{m}_{2}(\alpha)$ for some $\alpha \in \hat{\Delta}$ and $\lambda_{1}(\operatorname{Re} T)>\lambda_{2}(\operatorname{Re} T)$. Thus

$$
\begin{aligned}
& \mathbf{m}_{1}(\alpha) \lambda_{1}(\operatorname{Re} T)+\mathbf{m}_{2}(\alpha) \lambda_{2}(\operatorname{Re} T)+\sum_{j=3}^{n} m_{j}(\alpha) \lambda_{j}(\operatorname{Re} T) \\
& >\mathbf{m}_{2}(\alpha) \lambda_{1}(\operatorname{Re} T)+\mathbf{m}_{1}(\alpha) \lambda_{2}(\operatorname{Re} T)+\sum_{j=3}^{n} m_{j}(\alpha) \lambda_{j}(\operatorname{Re} T)
\end{aligned}
$$

since $\left(\mathbf{m}_{1}(\alpha)-\mathbf{m}_{2}(\alpha)\right)\left(\lambda_{1}(\operatorname{Re} T)-\lambda_{2}(\operatorname{Re} T)\right)>0$. Notice that $(\mathbf{m}(\alpha))(12)$ is still a multiplicity vector, where (12) denotes the transposition in $S_{n}$. So $D_{K}(T)$ would have more than one eigenvalue. Thus $D_{K}(T)$ would be non-scalar, which is a contradiction. Applying the same arguments, we see that $\operatorname{Im} T$ is a scalar, and the result follows.

Suppose $D_{K}(T)$ is Hermitian. Then $\operatorname{Im} D_{K}(T)$ is the zero operator. By the previous argument, we see that $\operatorname{Im} T$ is a scalar and thus the zero operator.

Now, (a) and (b) follow from the fact that $\operatorname{Spec} \operatorname{Re} e^{i r} D_{K}(T) \subseteq W^{*}\left(\operatorname{Re} e^{i r} D_{K}(T)\right)$ for any $r \in[0,2 \pi)$.

THEOREM 5.2. Suppose $\chi$ is not of determinamt type, and $\alpha, \beta \in \mathbb{C}$. The following conditions are equivalent.

(a) $W^{*}\left(D_{K}(T)\right)=W\left(D_{K}(T)\right) \subseteq\{\alpha t+\beta: t \in \mathbb{R}\}$.

(b) $D_{K}(T)=\alpha \tilde{H}+\beta I$ for some Hermitian operator $\tilde{H}$.

(c) $T=\alpha H+(\beta / m) I$ for some Hermitian operator $H$.

Proof. The equivalence of (a) and (b) is well known. That (c) implies (b) is clear. Suppose $D_{K}(T)=\alpha \tilde{H}+\beta I$ for some Hermitian operator $\tilde{H}$. Consider two cases. (1) $\alpha \neq 0 . \quad D_{K}[(T-(\beta / m) I) / \alpha]=\left(D_{K}(T)-\beta I\right) / \alpha=\tilde{H}$ is Hermitian, since $D_{K}(I)=m I$. Thus, $[T-(\beta / m) I] / \alpha$ is Hermitian by Theorem 5.1 (b). Condition (c) 
follows. (2) $\alpha=0$. Then $D_{K}(T)=\beta l$ and $W\left(D_{K}(T)\right)=\{\beta\}$. By Theorem 5.1 (a), $T=\beta I / m$.

\section{Related topics}

Suppose $m \leq n$. Researchers also consider

$$
W_{\perp}^{*}(L)=\left\{\left(L v^{*}, v^{*}\right) \begin{array}{l}
v^{*}=v_{1} * \cdots * v_{m},\left\{v_{1}, \ldots, v_{m}\right\} \text { is } \\
\text { an orthonormal set in } V
\end{array}\right\} .
$$

Using (3.2) with $\alpha=(1,2, \ldots, m)$, so that $\mathbf{m}_{t}(\alpha)=1$ for all $t=1, \ldots, m$, we have the following.

THEOREM 6.1. Suppose $m \leq n$. Then

$$
W_{\perp}^{*}\left(D_{K}(T)\right)=\left\{\frac{\chi(e)^{2}}{|H|} \sum_{j=1}^{m}\left(T v_{j}, v_{j}\right):\left\{v_{1}, \ldots, v_{m}\right\} \text { is an orthonormal set in } V\right\} \text {. }
$$

Thus, $W_{\perp}^{*}\left(D_{K}(T)\right)$ is, up to a scalar, the $m$ th numerical range of $T$ as defined by Halmos [7].

Another generalization is

$\widetilde{W}^{*}(L):=\left\{\left(L\left(u_{1} * \cdots * u_{m}\right), u_{1} * \cdots * u_{m}\right): u_{1}, \ldots, u_{m}\right.$ are unit vectors in $\left.V\right\}$.

Clearly $W_{\perp}^{*}(L) \subseteq \widetilde{W}^{*}(L)$.

THEOREM 6.2. Suppose $1<m<n, H=S_{m}$, and $\chi$ is the alternating character. Then $\widetilde{W}^{*}\left(D_{K}(T)\right)$ equals the convex hull of $(1 / n !) W_{m}(T) \cup\{0\}$.

Proof. Assume $1<m<n$ and put $L=D_{K}(T)$. Let $\mu \in(1 / n !) W_{m}(T)$. By Theorem 6.1, $\mu \in W_{\perp}^{*}(L)$, so $\mu=\left(L x^{*}, x^{*}\right)$ where $x^{*}=x_{1} * \cdots * x_{m}$ with $x_{1}, \ldots, x_{m}$ orthonormal. Let $0 \leq t \leq 1$. Set $u_{1}=\sqrt{t} x_{1}+\sqrt{1-t} x_{2}$ and $u_{j}=x_{j}, j=2, \ldots, m$. Then $u^{*}=\sqrt{t} x^{*}$ and thus $t \mu=\left(L u^{*}, u^{*}\right) \in \widetilde{W}^{*}(L)$. Since $W_{m}(T)$ is convex [7], the convex hull of $(1 / n !) W_{m}(T) \cup\{0\}$ is contained in $\widetilde{W}^{*}(L)$.

Conversely, let $\mu \in \widetilde{W}^{*}(L)$. Then $\mu=\left(L x^{*}, x^{*}\right)$ with $x_{1}, \ldots, x_{m}$ unit vectors. If $x_{1}, \ldots, x_{m}$ are linearly dependent, then $x^{*}=0$ and $\mu=0$. Assume $x_{1}, \ldots, x_{m}$, are linearly independent and let $\left\{u_{1}, \ldots, u_{m}\right\}$ be an orthonormal basis of the linear span of $x_{1}, \ldots, x_{m}$. Then, for $i=1, \ldots, m$, we can write $x_{i}=\sum_{j=1}^{m} a_{i j} u_{j}$ with $a_{i j} \in \mathbb{C}$, and $x^{*}=(\operatorname{det} A) u^{*}$, where $A=\left(a_{i j}\right)$. So $\mu=|\operatorname{det} A|^{2}\left(L u^{*}, u^{*}\right)$. However $|\operatorname{det} A|^{2}=\operatorname{det}\left(A A^{*}\right)$, which is less than or equal to 1 by the Hadamard inequality [4], so $\mu$ is contained in the convex hull of $W_{\perp}^{*}(L) \cup\{0\}$. Since $W_{\perp}^{*}(L)=(1 / n !) W_{m}(T)$ by Theorem 6.1, we have the desired result. 
REMARK. As pointed out by the referee, it would be interesting to find a non-convex example of $W^{*}\left(D_{K}(T)\right)$.

\section{References}

[1] Y. H. Au-Yeung and N. K. Tsing, 'Some theorems on the numerical range', Linear Multilinear Algebra 15 (1984), 3-11.

[2] N. Bebiano, C. K. Li and J. da Providencia, 'Some results on the numerical range of a derivation', SIAM J. Matrix Anal. Appl. 14 (1993), 1084-1095.

[3] — 'Generalized numerical ranges of permanental compounds arising from quantum systems of bosons', Electron. J. Linear Algebra 7 (2000), 73-91.

[4] R. Bhatia, Matrix Analysis (Springer, New York, 1997).

[5] D. Z. Djokovic and T. Y. Tam, 'Some questions about semisimple Lie groups originating in matrix theory', Canad. J. Math. 46 (2003), 332-343.

[6] M. Goldberg and E. G. Straus, 'Elementary inclusion relations for generalized numerical ranges', Linear Algebra and Appl. 18 (1977), 1-24.

[7] P. R. Halmos, A Hilbert Space Problem Book (Springer-Verlag, New York, 1978).

[8] R. R. Holmes, 'Orthogonal bases of symmetrized tensor spaces', Linear Multilinear Algebra 39 (1995), 241-243.

[9] R. R. Holmes and T. Y. Tam, 'Symmetry classes of tensors associated with certain groups', Linear Multilinear Algebra 32 (1992), 21-31.

[10] I. M. Isaacs, Character Theory of Finite Groups (Academic Press, New York, 1976).

[11] G. James and A. Kerber, The Representation Theory of the Symmetric Group, Encyclopedia of Mathematics and Its Applications, Volume 16 (Addison-Wesley Publishing Company, 1981).

[12] G. James and M. Liebeck, Representations and Characters of Groups, Cambridge Mathematical Textbooks (Cambridge University Press, Cambridge, 1993).

[13] C. K. Li and T. Y. Tam, 'Operator properties of $T$ and $K(T)^{\prime}$, Linear Algebra and Appl. 401 (2005), 173-191.

[14] C. K. Li and N. K. Tsing, 'The numerical range of derivations', Linear Algebra and Appl. 119 (1989), 97-119.

[15] C. K. Li and A. Zaharia, 'Decomposable numerical range on orthonormal decomposable tensors', Linear Algebra and Appl. 308 (2000), 139-152.

[16] M. Marcus, Finite Dimensional Multilinear Algebra, Part I (Marcel Dekker, New York, 1973).

[17] - Finite Dimensional Multilinear Algebra, Part II (Marcel Dekker, New York, 1975).

[18] - 'Some combinatorial aspects of numerical range', Second International Conference on Combinatorial Mathematis (New York) Ann. New York Acad. Sci. 319 (1979), 368-376.

[19] - 'Ranges of derivations', manuscript, 1989.

[20] R. Merris, Multilinear Algebra (Gordon and Breach Science Publishers, Amsterdam, 1997).

[21] Y. T. Poon, 'Another proof of a result of Westwick', Linear Multilinear Algebra 9 (1980), 35-37.

[22] R. T. Rockafellar, Convex Analysis (Princeton University Press, Princeton, 1970).

[23] T. Y. Tam, 'Convexity of generalized numerical range associated with a compact Lie group', $J$. Austral. Math. Soc. 71 (2001), 1-10.

[24] N. K. Tsing, 'On the shape of the generalized numerical ranges', Linear Multilinear Algebra 10 (1981), 173-182.

[25] B. Y. Wang, Foundation of Multilinear Algebra, (in Chinese) (Beijing Normal University Press, 1985). 
[26] B. Y. Wang and M. P. Gong, 'A high symmetry class of tensors with an orthogonal basis of decomposable symmetrized tensors', Linear Multilinear Algebra 30 (1991), 61-64.

[27] R. Westwick, 'A theorem on numerical range', Linear Multilinear Algebra 2 (1975), 311-315.

Department of Mathematics and Statistics Auburn University

Auburn

Alabama 36849-5310

USA

e-mail: holmerr@auburn.edu tamtiny@auburn.edu
Department of Mathematics College of William and Mary PO Box 8795, Williamsburg Virginia 23187-8795 USA e-mail: ckli@math.wm.edu 
J. Aust. Math. Soc. 82 (2007) 